\title{
Rapid environmental analysis using molten salt fusion sample preparation
}

\author{
By C. E. Armenta ${ }^{1}$, E. R. Gonzales ${ }^{1, *}$, J. A. Herrera ${ }^{1}$, A. A. Plionis ${ }^{1,2}$ and D. S. Peterson ${ }^{1}$ \\ ${ }^{1}$ Chemistry Division of the Los Alamos National Laboratory, PO Box 1663, Los Alamos, NM 87545, USA \\ 2 National Security Technologies, PO Box 380, Suitland, MD 20752, USA
}

(Received December 1, 2009; accepted in revised form January 25, 2011)

\begin{abstract}
Molten salt fusion / Environmental Am method / Sample preparation / Pu mobile method /

Alpha spectroscopy / Actinide field method
\end{abstract}

\begin{abstract}
Summary. For the analysis of the isotopic composition of environmental samples, including transuranic materials there are many methods that exist. This paper describes the development of a high throughput method, which involves dissolving a soil into an aqueous matrix, producing a homogenous mixture, and separating radionuclides to enable the identification of specific isotopes. A standard method was modified via changes in oxidation, chemical exchange, decomposition, or rearrangements to form constituents that are more soluble in acidic aqueous solutions. To accomplish this, a molten-salt fusion, dissolution in dilutes nitric or hydrochloric acids, with subsequent separation using ion exchange, direct deposition, and counting by alpha-spectrometry was used. This method is ideal for silicate samples, but can be modified to accommodate more complex soil samples.
\end{abstract}

\section{Introduction}

Standard methods for actinide separation analysis are used for environmental samples and yield high quality data [1]. They are especially favorable because they produce a homogenized mixture and result in total destruction of the sample. However, these standard methods are labor and time intensive, use hazard chemicals, and require extensive amount of equipment. Because of these issues, we have evaluated two new methods, a rapid and a rapid mobile method. Both can be considered rapid turnaround methods that have great potential to be used on environmental samples. The rapid mobile method has been successfully applied to both simple (silicate) and complex Environmental Measurements Laboratory (Quality Assurance Program QAA certified samples QAP) soil samples.

The sample dissolution process is the biggest challenge that the analytical chemist must overcome when working with difficult types of matrices, due to the fact that samples are made of unknown compounds with unknown chemistries [2]. It is very important when the chemist commences work on a project that they have met with the cus-

\footnotetext{
*Author for correspondence (E-mail: ed_gonzales@lanl.gov).
}

tomer to ensure that the objectives are clearly known prior to sample processing. With this in mind the chemist must define the data parameters for bias and precision so as to fulfill the data quality objectives of the program. There are some questions that may need to be considered prior to processing, what is the nature of the sample to be analyzed? Is it refractory or is the contamination on the surface of the sample? How effective is the dissolution and how much of the target can be lost and still be of value? Many times the customer is satisfied with quantitative information and some customers require qualitative data with supporting quality parameters. These and other factors must be balanced before the best method can be chosen. Fusion, high temperature molten salt digestion, is the best method when dissolution of a difficult matrix is desired in a limited amount of time. Many fusions can be accomplished from fusion to dissolution in less than an hour [2,3]. Fusion offers the best possible alternative to classic radiochemical analysis when the intent is a rapid method.

The need for rapid methods for isotopic identification of actinides in silicon-based matrices has become a major factor as emergency analysis becomes the overriding concern. Unique circumstances require a rapid method capable of separation with identification of select nuclear isotopes. The method needs to be robust enough to accommodate a fused silicon matrix, yet simple enough to be performed in the field under less than ideal conditions. Depending on matrix characteristics the sample may have to be completely dissolved before isotopic identification is possible. Ideally direct leach, placing the sample in a concentrated acid, would be the preferred method due to the simplicity of the method, but surface contamination may not be sufficient to warrant direct leaching, more qualitative information about the total sample may be needed, therefore direct leaching is an undesirable method to give information about the total sample. That is why fusion is the preferred method.

\section{Experimental}

\subsection{Materials and instruments}

Reagents used were prepared using analytical grade chemicals. Sodium nitrite, hydrochloric acid, sulfuric acid, ammonium hydroxide, thymol blue indicator, and nitric acid 
were obtained from Fisher Scientific (Pittsburgh, PA). NIST traceable plutonium-242 and americium-243 standards were obtained from Isotope Products Laboratories (Valencia, CA) and Analytics Laboratory (Atlanta, GA). These isotopes were selected so that they could be analyzed in the alpha spectrometer as tracer standard addition with Environmental Measurements Laboratory (EML) QAP - 59 samples. Although EML is no longer operational the QAP are used as traceable certified materials. Deionized water was used for solution preparation. Separations were performed using Bio Rad resin AG-1X4, chloride form, 50-100 mesh supported with a glass wool plug; Eichrom TRU cartridges and Bio Rad borosilicate columns. Transuranic separations were performed using Eichrom Vacuum Box. Samples were plated onto 3/4 inch stainless steel disks on an electro deposition unit designed at LANL. Counting was performed on an Ortec (Oak Ridge, TN) Octete Plus Alpha Spectrometer. The alpha spectrometers were equipped with $450 \mathrm{~mm}$ PIPS detectors with chamber settings providing counting efficiencies of up to $30 \%$.

\subsection{Selection of fusion salt}

Many experiments were preformed on different types of salts prior to running samples. Presented are the selected salts that were fused with sand at a temperature about $100^{\circ} \mathrm{C}$ above the melting point of the salt. These samples were initially fused in a preheated muffle furnace. Initial tests helped eliminate the following salts from consideration; lithium metaborate, lithium tetraborate, eutectic mixtures of lithium metaborate and lithium tetraborate, lithium carbonate, lithium fluoride, mixtures of lithium fluoride and lithium metaborate, mixtures of lithium fluoride and lithium tetraborate, and boric acid. These salts were fused and produced what appeared to be high quality melts, but were hard to dissolve either in water, dilute nitric, or hydrochloric acids. The salts with the best end point, this being ease of dissolution, were sodium hydroxide, potassium hydroxide, and sodium carbonate. Sodium carbonate required a fusion temperature of $1000{ }^{\circ} \mathrm{C}$ and an hour or more to dissolve. Sodium and potassium hydroxide fused at about $600{ }^{\circ} \mathrm{C}$ and required 20 minutes to dissolve. Sodium and potassium hydroxide could also be fused over the open flame produced by a Bunsen burner. Ultimately the salt of choice for the mobile rapid method is sodium hydroxide due to the desired characteristics that is needed in a rapid method, using a Bunsen burner and ease of dissolution after melt has occurred.

\subsection{Ion exchange separations}

The process for these separations is detailed in Fig. 1. The diagram on the left sides depicts separation using the Bio$\mathrm{Rad}$ column and is used to separate Pu from the matrix and other interfering elements. This procedure is versatile and can accommodate different samples depending on isotopes of interest and is routinely used for $\mathrm{Pu}$ and $\mathrm{U}$ separations [4]. The BioRad column can accommodate both $\mathrm{HNO}_{3}$ and $\mathrm{HCl}$ acids. The reason Fig. 1 diagram depicts the acid type and concentration as XM is because the process is dependent on isotope of interest on the concentration of the acid that is being used, it can vary so a generic diagram is shown. The right side diagram illustrates separations with the Eichrom vacuum box procedure and is used to isolate Am from the sample [5-7]. The vacuum box can accommodate single cartridges or can be used in tandem with other cartridges for multiple separations running together. The vacuum box can also be operated in gravity mode requiring no vacuum.

\subsection{Plating}

Electro deposition and stippling were performed on all samples. The electro deposition used was a modification of the procedure developed by Talvite [8] and utilizes an ammonium sulfate electrolyte with a plating current of $0.55 \mathrm{Amps}$ and plating time of between 2-3 h depending on isotope of interest. Stippling utilized a flat bottom alpha beta planchet of 1-inch diameter. Stippling is a method, in which we concentrate the clean ion exchanged eluent the planchet is
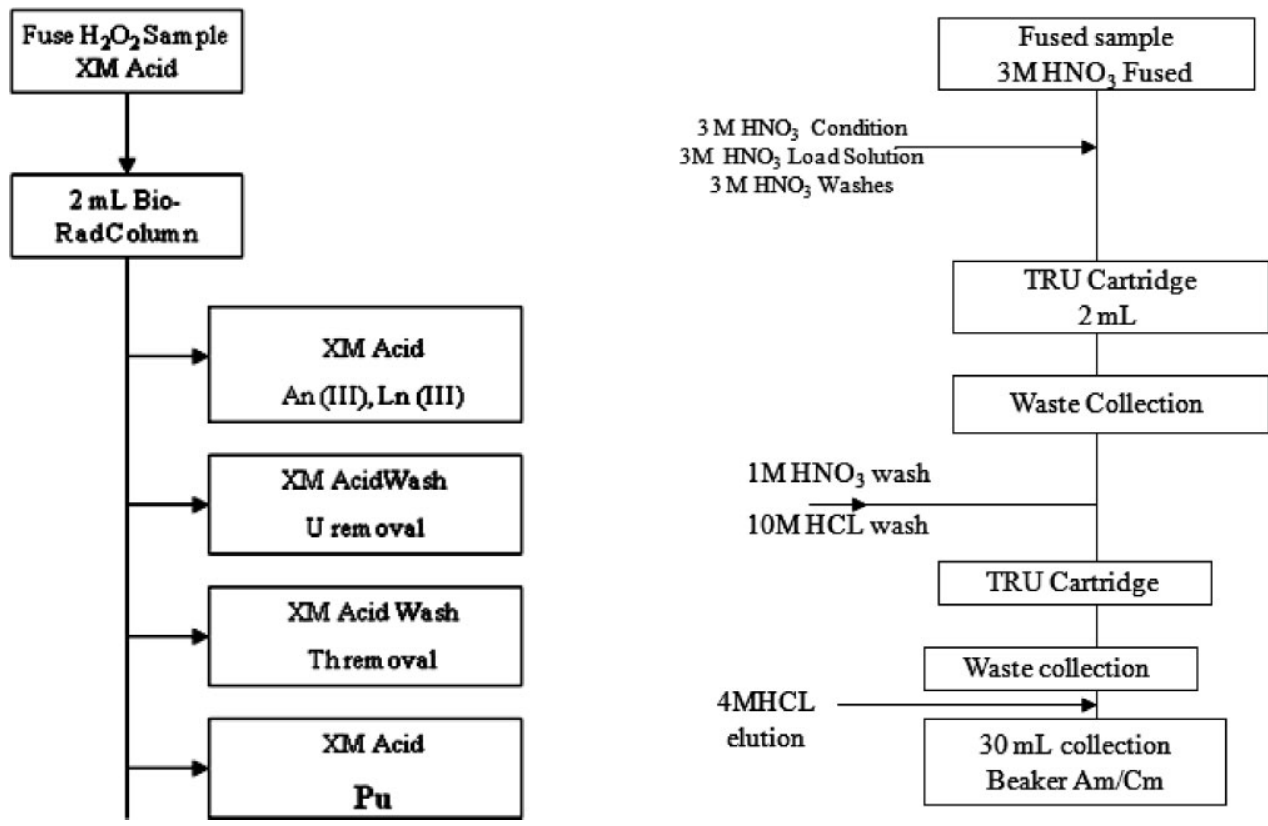

Fig. 1. Analytical separation diagram. Left diagram the XM designates possible use of either nitric or hydrochloric acid at various concentrations. General depiction of a separation technique. 
placed on the surface of the hot plate, then the eluent is direct deposited and dried on the surface of the planchet fixing the isotope of interest on the planchet.

\section{Results and discussion}

The sample chosen for analyses was from an area where a vitrification process was employed to immobilize metals. The sample contained trace quantities of plutonium, americium, and uranium. Vitrified samples from this location had been analyzed previously and data was collected to establish the baseline concentration of the isotopes required. Prior to fusion, $2 \mathrm{~g}$ of the sample was analyzed by standard analytical procedures to validate activity levels. Samples were processed by HF digest [2]. The Standard Method data correlated well with the previously generated data from another organization but in this case the standard method data were considered as certified for these comparisons. This data was then compared to data generated by fusion preparation methods found in Table 1. The generated data indicated that all of the target isotopes were present in the fused samples. Error propagation was limited to counting error, sample weighing, tracer uncertainty (NIST Traceable), and tracer pipetting. Sample homogeneity was not accounted for.

A direct leach was also performed and the data indicated that the target isotopes were bound to the silicate structure of the sample as indicative by the low recovery of the target isotopes even after correction for tracer addition.

Data generated from the sodium carbonate fusion after tracer corrections were equal to the tracer corrected data from the HF digest found in Table 2. Separation was clean with little interference, the eluent was clean with no signs of salts or matrix in the eluent, and there were no interferences or salt build up in the plating solution that would plate out and broaden the peaks of the spectra. Isotopes and cor-
Table 1. Methods table: description and time of selected methods.

\begin{tabular}{llc}
\hline & Description & Time (d) \\
\hline Standard method & HF digest & 7.00 \\
\hline Rapid method & Bicarbonate fusion & 2.00 \\
\hline Rapid mobile method 1 & $\begin{array}{l}\mathrm{NaOH} \text { fusion } \\
\mathrm{HCl}-\mathrm{HCl}_{2} \text { separation } \\
\text { Electro-deposition }\end{array}$ & 0.42 \\
\hline Rapid mobile method 2 & $\begin{array}{l}\text { NaOH fusion } \\
\mathrm{HNO}_{3} \text { separation } \\
\mathrm{Electro}^{-d e p o s i t i o n}\end{array}$ & 0.33 \\
\hline Rapid mobile method 3 & $\begin{array}{l}\mathrm{NaOH} \text { fusion } \\
\mathrm{HNO}_{3} \text { separation } \\
\mathrm{Stippling}\end{array}$ & 0.25 \\
\hline
\end{tabular}

responding activities were accounted for in each separate ion exchange performed. Although the melt required additional mechanical (heating and stirring) work to get it to dissolve, the data generated with the carbonate method was equivalent to that generated by the standard method. The advantage to using the carbonate fusion was that, it reduced the time required for sample preparation to about $1 \mathrm{~d}$ and total separation time down to two days. For this reason the method is designated as the Rapid method and method times are reported in Table 1. Because these samples had relatively low concentrations of the target isotopes, standard count time of $24 \mathrm{~h}$ was used. Our primary objective was to ensure that the fusion method would be able to solubilize bound isotopes. All target isotopes $(\mathrm{Pu}, \mathrm{Am}$ and $\mathrm{U})$ were recovered at 80 $100 \%$ as determined by recovered tracer. In all cases the HF digest data generated by the Standard Method are considered the Reference Data. It is important to note that a standard HF digestion can take up to $4 \mathrm{~d}$ to perform.

Table 2. Comparison table: vitrified sample analyses data units $\mathrm{pCi} / \mathrm{g}$.

\begin{tabular}{lcclcccc}
\hline $\begin{array}{l}\text { Sample } \\
\text { ID }\end{array}$ & Method & Pu-239 & Error & Pu-238 & Error & MDA & $\begin{array}{c}\text { Tracer } \\
\% \text { rec }\end{array}$ \\
\hline Split 1 & Fusion \#1 & 113.4 & 1.16 & 0.536 & 0.0135 & 0.1 & 100 \\
Split 2 & Fusion \#2 & 114.6 & 1.17 & 0.559 & 0.0138 & 0.2 & 79 \\
Split 3 & Fusion \#3 & 72.7 & 0.75 & 0.429 & 0.0119 & 0.1 & 103 \\
Split 4 & Fusion \#4 & 69.5 & 0.71 & 0.175 & 0.0139 & 0.1 & 99 \\
Split 5 & HF digest & 113.2 & 1.15 & 0.520 & 0.0135 & 0.1 & 96 \\
Split 6 & Nitric leach & 0.22 & 0.008 & 0.22 & 0.008 & 0.2 & 96 \\
\hline Sample & Method & U-238 & Error & U-234 & Error & MDA & Tracer \\
ID & & & & & & & $\%$ rec \\
\hline Split 3 & Fusion \#3 & 3.23 & 0.045 & 5.54 & 0.069 & 0.2 & 90 \\
Split 4 & Fusion \#4 & 3.42 & 0.039 & 5.66 & 0.070 & 0.2 & 100 \\
Split 5 & HF digest & 5.94 & 0.072 & 9.46 & 0.109 & 0.1 & 100 \\
Split 6 & Nitric leach & 0.074 & 0.016 & 0.033 & 0.010 & 0.1 & 100 \\
\hline Sample & Method & Am-241 & Error & MDA & Tracer & & \\
ID & & & & & $\%$ rec & & \\
\hline Split 3 & Fusion \#3 & 2.43 & 0.036 & 0.2 & 90 & & \\
Split 4 & Fusion \#4 & 2.51 & 0.037 & 0.1 & 92 & & \\
Split 5 & HF digest & 2.45 & 0.036 & 0.1 & 100 & & \\
Split 6 & Nitric leach & 0.077 & 0.002 & 0.2 & 78 & & \\
\hline & & & & & & & \\
\hline
\end{tabular}


Data generated from the sodium hydroxide fusion $(2 \mathrm{~g}$ sample to $10 \mathrm{~g} \mathrm{NaOH}$ ) was not equivalent to that generated by the standard method. This method is designated Rapid Mobile Method. The experimental objective was a nontraced rapid field deployable method suitable for isotope identification. Although tracer studies (not shown) utilizing this method showed equivalency to the Standard Method our intent was a non-traced method. The $\mathrm{NaOH}$ fusion $(2 \mathrm{~g}$ sample to $10 \mathrm{~g} \mathrm{NaOH}$ ) was prepared over an open flame and was complete in about $15 \mathrm{~min}$, the dissolution of the melt takes an additional $20 \mathrm{~min}$ to dissolve the sample for separation. The separations were not as clean as those for the carbonate fusion. The ion exchange data indicated a carry over of plutonium into the uranium separation with about $40 \%$ of the bound $\mathrm{Pu}$ ending up with the uranium. The UTEVA uranium procedure used does not discriminate against the $\mathrm{Pu}$ [7]. and isotopic equilibration did not occur in the time between fusion and added tracer. In this study tracers were added after the fusion prep. This information is very important, as there are several things that can be done to the fusion process to insure proper oxidation states. The calculated amount of plutonium in the plutonium separation and uranium separation accounted for all the plutonium in the sample. The tracer corrected americium is quantitative which is understandable, since Am exhibits only one oxidation state in this process. Total uranium was not quantitative and may indicate sample was lost during fusion. Since the sample was traced after fusion the tracer corrects for losses during ion exchange. Further investigation may be required. Unfortunately uranium and americium were not determined for the carbonate fusion. It is possible that we are seeing a lack of homogeneity in the analyzed sample.

In creating these new methods, we sought to compare the maximum time taken for completion to that of the established standard method. The standard method takes seven days to complete. When using the Rapid Method, the total time was significantly decreased by five days. We were able to further decrease the total time with the variations of the Rapid Mobile Method. All three of these variations methods took less than one day to complete. Rapid Mobile Method 1, which used an $\mathrm{HCl}$ separation and electro-deposition, had a completion time of ten hours. When a $\mathrm{HNO}_{3}$ separation was substituted as in Rapid Mobile Method 2, $8 \mathrm{~h}$ was needed to complete the entire process. Finally, in using a $\mathrm{HNO}_{3}$ separation and stippling, the deposit method previously mentioned, the maximum time needed for completion was reduced to only $6 \mathrm{~h}$. Table 2 summarizes these results. It must be noted that the standard method has been used with samples up to $100 \mathrm{~g}$ in size. This is not possible with the Rapid Methods which has severe limitations after $2 \mathrm{~g}$.

Aside from examining the time required for completion, it was also necessary to determine the quality of data produced by each method. To do this, measurements for plutonium and americium from each method were divided by certified values defining our Figure of Merit. The closer the ratio was to one the higher the quality of data the method produced. Ratios were first determined using vitrified samples. The certified values for $\mathrm{Pu}-238, \mathrm{Pu}-239$, and $\mathrm{Am}-$ 241 were $0.52 \mathrm{pCi} / \mathrm{g}, 113 \mathrm{pCi} / \mathrm{g}$, and $2.5 \mathrm{pCi} / \mathrm{g}$ respectively. The standard method was defined as the certified method for the isotopes of Pu-238, Pu-239, and Am-241. When the data
Table 3. Summary table showing ratio (measured/certified) for vitrified soil samples (standard method is set as certified value).

\begin{tabular}{lcccc}
\hline Isotopes & $\begin{array}{c}\text { Rapid } \\
\text { method }\end{array}$ & $\begin{array}{c}\text { Rapid } \\
\text { mobile } \\
\text { method 1 }\end{array}$ & $\begin{array}{c}\text { Rapid } \\
\text { mobile } \\
\text { method 2 }\end{array}$ & $\begin{array}{c}\text { Rapid } \\
\text { mobile } \\
\text { method 3 }\end{array}$ \\
\hline $\mathrm{Pu}-238$ & $1.03 \pm 0.034$ & $1.07 \pm 0.033$ & $0.82 \pm 0.036$ & $0.34 \pm 0.083$ \\
$\mathrm{Pu}-239$ & $1.01 \pm 0.028$ & $1.01 \pm 0.027$ & $0.64 \pm 0.027$ & $0.61 \pm 0.026$ \\
$\mathrm{Am}-241$ & - & - & $0.99 \pm 0.031$ & $1.02 \pm 0.030$ \\
\hline
\end{tabular}

from the Rapid Method was divided by the standard method it produced ratios very close to 1.00 . The variations of the Rapid Mobile methods produced ratio as high as 0.99 and as low as 0.34 . Table 3 summarizes the resulting ratios for the vitrified samples.

It was determined that the vitrified sample does not adequately represent real field sample due to the lack of organic material. The vitrified sample has more of a glassy composition. EML QAP samples have more organics and interfering material and better represent real samples, therefore the EML sample was chosen to analyze and compare data. Normally EML samples are analyzed at a $10 \mathrm{~g}$ sample size. Homogeneity problems occur when samples less than $10 \mathrm{~g}$ are used but fusions do not work well above $2 \mathrm{~g}$. All methods were normalized to $2 \mathrm{~g}$ for analyses and proved to be a substantially harder sample to analyze. Since QAP samples are also used as an inorganic and organic certified material, these added components required extra vigilance in the fusion process and required an extra step. Samples required muffling at $700{ }^{\circ} \mathrm{C}$ for one hour or fusion time had to be doubled. Ratios were then calculated for the QAP 59 soil samples. The certified values are as follows: $0.395 \pm$ $0.046 \mathrm{pCi} / \mathrm{g}$ for $\mathrm{Pu}-238,0.822 \pm 0.081 \mathrm{pCi} / \mathrm{g}$ for $\mathrm{Pu}-239$ and $0.497 \pm 0.048 \mathrm{pCi} / \mathrm{g}$ for Am-241. Table 4 presents the calculated ratios. The ratios produced from standard method data were slightly above 1.00 , while the ratios produced from the rapid mobile method data ranged from 0.73 to 1.1 . It is possible that variations in the methods could be attributed to lack of sample homogeneity, but assessing this is beyond the scope of the methods studied.

In order to improve the process time, samples were stippled instead of electroplated onto a 1-inch standard al$\mathrm{pha} / \mathrm{beta}$ planchet to determine if the 2-h plating time could be replaced. The sample run through the Bio Rad resin seemed amenable to stippling with little residue on the planchet surface. Samples run through the Eichrom resins deposited small quantities of material, which we presumed was some of the extractive ligand. Our routine analyses with TRU resins always showed evidence of material in the plating solutions. This material does not present a problem for

Table 4. Summary table showing ratio (measured/certified) for QAP soil samples (QAP-59 data set as certified data).

\begin{tabular}{llll}
\hline Isotopes & $\begin{array}{c}\text { Standard } \\
\text { method }\end{array}$ & $\begin{array}{c}\text { Rapid mobile } \\
\text { method 1 }\end{array}$ & $\begin{array}{c}\text { Rapid mobile } \\
\text { method 2 }\end{array}$ \\
\hline $\mathrm{Pu}-238$ & $1.15 \pm 0.13$ & $0.90 \pm 0.09$ & $1.08 \pm 0.11$ \\
$\mathrm{Pu}-239$ & $1.20 \pm 0.11$ & $0.97 \pm 0.096$ & $0.73 \pm 0.069$ \\
$\mathrm{Am}-241$ & $1.04 \pm 0.10$ & $1.00 \pm 0.10$ & $0.73 \pm 0.12$ \\
\hline
\end{tabular}


electro deposition but did affect resolution of the stippled sample for alpha spectroscopy with Full Width Half Max (FWHM) at over $200 \mathrm{keV}$. Plated samples average FWHM of $30 \mathrm{keV}$. Compensating for this peak broadening the calculated recoveries were acceptable and the procedure time was reduced to $4 \mathrm{~h}$, (Table 1 ).

\section{Conclusions}

We have shown the potential for a Rapid Mobile Procedure. This method utilizes $\mathrm{NaOH}$ fusion, modified chemistry, with stippling capable of quantitative determination of actinides in the field with a $6-\mathrm{h}$ process time and minimal chemicals. We have also demonstrated the potential of a Rapid Method equivalent to Standard methods with a process time of 1-2 d, if sample size is not a major consideration. The data obtained demonstrate fast procedures with the capability to increase through put of samples in an emergency situation where analysis in the field is possible.

Process times of $6 \mathrm{~h}$ to $48 \mathrm{~h}$ are significant improvements over typical lab based methods and show the potential for the development of stand-alone field methods. Small improvements in radiation count technology would provide for the basis of a completely portable alpha spectroscopy analysis procedure. Modification of sample preparation and separations are the first step in this development. These methods are a significant improvement over classical wet radiochemical sample preparation with potential for wide application. Future studies will examine the effects of modification to the $\mathrm{NaOH}$ and carbonate flux mechanism to enhance oxidation state and equilibrium reactions of the samples, which in turn could increase the yields with better separations techniques. Modification to the process can provide a cleaner separation allowing for a better-stippled sample. We will also further evaluate new technologies with the potential to replace electro-deposition and stippling completely setting the stage for analysis and counting in 6-8 h.

Acknowledgment. The Los Alamos National Laboratory is operated by Los Alamos National Security LLC, for the US Department of Energy under Contract number DE-AC52-06NA25396. Funding through Los Alamos National Laboratory Analytical Chemistry Emergency Capability is gratefully acknowledged. This publication is LAUR-09-07757.

\section{References}

1. Sill, C. W.: some problems in measuring plutonium in the environment 1975. Health Phys. 29, 619 (1975).

2. Bock, R.: A Handbook of Decomposition Methods in Analytical Chemistry. T. \& A. Constable Ltd., Edinburgh (1979), p. 86.

3. US Environmental Protection Agency: Multi-Agency Radiological Laboratory Analytical Protocols July 2004 (2004), p. 13.

4. Veselsky, J. C.: Problems in the determination of plutonium in bioassay and environmental samples. Anal. Chim. Acta 90, 1 (1977).

5. Horwitz, P. E., Dietz, M. L., Chiarizia, R., Diamond, H.: Separation and preconcentration of uranium from acidic media by extraction chromatography. Anal. Chim. Acta 266, 25 (1992).

6. Eichrom Technologies Inc.: Analytical Procedures ACW03 VBS Rev. 1.6, 1 (2005).

7. Eichrom Technologies Inc.: Analytical Procedures ACS07 Rev. $1.5,1$ (2005) 